\title{
Design and testing of a dynamically-tuned magnetostrictive spring with electrically-controlled stiffness
}

\author{
Justin J. Scheidler $^{a}$, Vivake M. Asnani ${ }^{b}$, Marcelo J. Dapino ${ }^{a}$ \\ ${ }^{a}$ NSF I/UCRC on Smart Vehicle Concepts, Department of Mechanical and Aerospace \\ Engineering, The Ohio State University, Columbus, OH, USA, 43210 \\ ${ }^{b}$ Rotating and Drive Systems Branch, Materials and Structures Division, NASA Glenn \\ Research Center, Cleveland, OH 44135
}

\begin{abstract}
This paper details the development of an electrically-controlled, variable-stiffness spring based on magnetostrictive materials. The device, termed a magnetostrictive Varispring, can be applied as a semi-active vibration isolator or switched stiffness vibration controller for reducing transmitted vibrations. The Varispring is designed using 1D linear models that consider the coupled electrical response, mechanically-induced magnetic diffusion, and the effect of internal mass on dynamic stiffness. Modeling results illustrate that a Terfenol-D-based Varispring has a rise time almost an order of magnitude smaller and a magnetic diffusion cut-off frequency over two orders of magnitude greater than a Galfenol-based Varispring. The results motivate the use of laminated Terfenol-D rods for a greater stiffness tuning range and increased bandwidth. The behavior of a prototype Varispring is examined under vibratory excitation up to $6 \mathrm{MPa}$ and $25 \mathrm{~Hz}$ using a dynamic load frame. For this prototype, stiffness is indirectly varied by controlling the excitation current. Preliminary measurements of continuous stiffness tuning via sinusoidal currents up to $1 \mathrm{kHz}$ are presented. The measurements demonstrate that the Young's modulus of the Terfenol-D rod inside the Varispring can be continuously varied by up to $21.9 \mathrm{GPa}$. The observed stiffness tuning range is relatively constant up to 500 $\mathrm{Hz}$, but significantly decreases thereafter. The stiffness tuning range can be greatly increased by improving the current and force control such that a more consistent current can be applied and the Varispring can be accurately tested at a more optimal bias stress.
\end{abstract}

Keywords: dynamic stiffness tuning, magnetostrictive materials, Galfenol, Terfenol-D, vibration control

\section{INTRODUCTION}

Magnetostrictive materials, such as Galfenol $\left(\mathrm{Fe}_{1-x} \mathrm{Ga}_{x}, 0.13 \leq x \leq 0.29\right)$ and Terfenol-D $\left(\mathrm{Tb}_{x} \mathrm{Dy}_{1-x} \mathrm{Fe}_{y}\right.$, $x \approx 0.3, y \approx 2$ ), can transduce energy between magnetic and mechanical domains, thereby providing actuation and sensing effects. These materials also exhibit a variety of secondary effects, including stress- and magnetic field-dependent elastic moduli, which result from the superposition of purely elastic strain and magnetoelastic strain. ${ }^{1}$ This effect can be controlled precisely and remotely using electromagnets and can be harnessed for many applications, such as impedance matching, tunable mechanical resonators, ${ }^{2}$ semi-active vibration absorbers, ${ }^{3-6}$ and switched stiffness vibration control. ${ }^{7,8}$ Variations in elastic moduli with static changes in the bias magnetic field (termed the $\Delta E$ effect) have been well documented in Terfenol- $\mathrm{D}^{2,9-12}$ and Galfenol. ${ }^{13-18}$ To realize switched stiffness vibration control and real-time stiffness tuning, dynamic tuning of the elastic properties must be understood. However, dynamic tuning has not been investigated to date.

In this paper, a magnetostrictive transducer is designed to operate as a spring element that has a dynamically-tunable and electrically-controllable stiffness. This device is referred to as a magnetostrictive Varispring. The Varispring is designed by modeling (a) the coupled electrical response using a linear

Further author information: (Send correspondence to M.J.D)

J.J.S.: E-mail: scheidler.8@osu.edu, Telephone: 1-614-247-7480

M.J.D.: E-mail: dapino.1@osu.edu, Telephone: 1-614-688-3689

Industrial and Commercial Applications of Smart Structures Technologies 2015,

edited by Kevin M. Farinholt, Steven F. Griffin, Proc. of SPIE Vol. 9433, 94330F

(C) 2015 SPIE · CCC code: $0277-786 X / 15 / \$ 18 \cdot$ doi: 10.1117/12.2085574

Proc. of SPIE Vol. $943394330 \mathrm{~F}-1$ 
transducer model, (b) the effect of dynamic stress on bias magnetic fields (mechanically-induced magnetic diffusion), and (c) the effect of internal mass on the dynamic stiffness of the active element. First, experimental and practical design considerations are discussed. Then, the theoretical performance of Galfenoland Terfenol-D-based Varisprings are calculated and compared, after which the Varispring design is introduced. A prototype Varispring is manufactured and preliminary measurements of its dynamic stiffness tuning performance are presented.

\section{MODELING AND DESIGN}

\subsection{Experimental and Practical Considerations}

Future performance testing of the magnetostrictive Varispring will involve using the device in an experiment to simulate the variable stiffness of certain machine components. To facilitate this future experiment, the following design constraints were imposed on the prototype Varispring: (a) an axial stiffness of about 500 $\mathrm{N} / \mu \mathrm{m}\left(2.86 \times 10^{6} \mathrm{lbf} / \mathrm{in}\right)$, (b) a maximum applied dynamic force of $1000 \mathrm{~N}(224.8 \mathrm{lbf})$, and (c) a maximum diameter and height of $50 \mathrm{~mm}$ (1.97 in) and $105 \mathrm{~mm}$ (4.13 in), respectively. For precise stiffness tuning, it is desirable to operate the magnetostrictive material under small amplitude dynamic stresses, such that the material's response is approximately linear for a fixed magnetic input. Also, since the performance of Terfenol-D is slightly degraded above room temperature ${ }^{2}$ air cooling of the magnetostrictive rod is beneficial; this necessitates an air gap between the electromagnet and magnetostrictive rod. Consequently, the length of the magnetostrictive rod was used as an independent design variable, while the rod's diameter and the electromagnet's maximum dimensions were defined by the aforementioned criteria.

As discussed in detail by Scheidler et al., ${ }^{18}$ the vibration of any mass located in-between the specimen (i.e., the magnetostrictive rod) and force transducer introduces errors in the measurement of the dynamic force in the specimen; this inertial force error is reduced by minimizing the mass in-between the specimen and force transducer. To meet the ASTM-recommended inertial force error tolerance of $0.5 \%,{ }^{19}$ the dynamic, axial force applied to the specimen was measured by a piezoelectric load washer that was located inside the Varispring and nearly adjacent to the specimen.

To increase the changes in stiffness and operate in quasi-linear regimes, a mechanical preload was applied to the magnetostrictive rod. Typically, the preload is applied by a softening Belleville spring operated near its maximum deflection to prevent excessive preload variation during loading of the device. ${ }^{2}$ Thus, the stiffness of the Varispring's central load path relative to that of its magnetic flux return path was considered to ensure that the Belleville spring compresses during assembly of the device.

\subsection{Electrical Response}

For excitation frequencies at which internal dynamics can be neglected, the electromechanical response of a magnetostrictive transducer can be described by the following lumped parameter model, ${ }^{20,21}$

$$
\begin{aligned}
& F(s)=\frac{K^{H}}{s} \Delta v(s)-G I(s), \\
& V(s)=G \Delta v(s)+\left(L^{S} s+R_{\text {coil }}\right) I(s),
\end{aligned}
$$

where $s$ is the Laplace parameter, $F$ represents the force in the magnetostrictive rod (tension positive), $K^{H}$ is the axial stiffness of the rod at constant magnetic field, $\Delta v$ denotes the relative velocity of the ends of the rod, $I$ and $V$ are the current in and voltage applied to the electromagnet, respectively, and $R_{\text {coil }}$ is the electromagnet's resistance. Eqs. 1 and 2 are valid for linear constitutive regimes. The inductance at constant strain (blocked inductance) $L^{S}$ is

$$
L^{S}=\frac{N^{2} A_{\text {coil }} \mu^{S}}{l_{\text {coil }}}=\frac{N^{2} A_{\text {coil }}\left(\mu^{T}-d^{2} E^{H}\right)}{l_{\text {coil }}},
$$


Table 1: Material properties used for modeling the Varispring.

\begin{tabular}{c|c|c|c|c|c|c|c}
\hline Material & $\mu^{T} / \mu_{0}$ & $d(\mathrm{~nm} / \mathrm{A})$ & $E_{\text {sat }}(\mathrm{GPa})$ & $E_{\min }^{H}(\mathrm{GPa})$ & $\sigma(\mathrm{S} / \mu \mathrm{m})$ & $\rho\left(\mathrm{kg} / \mathrm{m}^{3}\right)$ & $H_{\max }(\mathrm{kA} / \mathrm{m})$ \\
\hline Galfenol & 250 & 40 & 71 & 33 & 5.88 & 7870 & 16 \\
Terfenol-D & 3 & 5 & 108 & 18 & 1.72 & 9250 & 70 \\
\hline
\end{tabular}

where $\mu^{T}$ (or $\mu^{S}$ ), $d$, and $E^{H}$ denote the magnetic permeability at constant stress (or strain), piezomagnetic coefficient, and Young's modulus at constant field of the magnetostrictive rod, respectively, $N$ and $l_{\text {coil }}$ are the number of windings in and axial length of the electromagnet, respectively, and $A_{\text {coil }}$ is the cross-sectional area enclosed by the electromagnet. ${ }^{20}$ The electromechanical coupling coefficient $G$ is

$$
G=\frac{N d A_{\text {rod }} E^{H}}{l_{\text {coil }}},
$$

where $A_{\text {rod }}$ is the cross-sectional area of the magnetostrictive rod. ${ }^{20}$ Assuming a mechanical loading of mass $m$, Eqs. 1 and 2 can be combined to determine the electrical response of the Varispring in terms of the effective electrical impedance $Z_{\mathrm{e}}$,

$$
V(s)=Z_{\mathrm{e}} I(s)=\left[\left(L^{S} s+R_{\text {coil }}\right)+\frac{G^{2} s}{m s^{2}+K^{H}}\right] I(s) .
$$

Assuming that magnetic flux leakage and the magnetic reluctance of the Varispring's flux return path are negligible, the magnetic field in the magnetostrictive rod is $H(s)=N I(s) / l_{\text {coil }}$. Insertion of Eq. 5 into the expression for $H(s)$ gives

$$
H(s)=\frac{N}{l_{\text {coil }}} \frac{m s^{2}+K^{H}}{L^{S} m s^{3}+R_{\text {coil }} m s^{2}+\left(G^{2}+L^{S} K^{H}\right) s+R_{\text {coil }} K^{H}} V(s) .
$$

Note that this model does not include eddy current effects, and is thus more accurate for finely laminated magnetostrictive rods.

The Varispring was operated at a mechanical bias for which the zero-field elastic state was stiff. Stiffness was reduced by increasing the field from zero to the field for which stiffness was minimized, herein termed the maximum tuning field $H_{\max }$. To approximate the response time of the Varispring to a step change in the command stiffness, the time required to increase the field from zero to $H_{\max }$ (i.e., the rise time) was calculated as a function of the magnetostrictive rod's length by solving Eq. 6 in Simulink for a step voltage $V(s)=V_{0} / s$. The material properties used in the simulations are given in Table 1. For a given geometry of the electromagnet, the gauge of wire it is wound with determines the maximum number of windings $N_{\max }$ and current $I_{\max }$. Rise times were calculated using the minimum number of windings needed to generate the maximum tuning field with $95 \%$ of the maximum current, i.e., $N_{\min }=H_{\max } l_{\text {coil }} /\left(0.95 I_{\max }\right)$. In this way, the effective electrical inductance was minimized for each design case. The rise times for Galfenol- and Terfenol-D-based Varisprings are shown in Fig. 1a.

For a given wire gauge, a Terfenol-D-based Varispring responds almost an order of magnitude faster than a Galfenol-based Varispring, despite the Terfenol-D requiring a much larger tuning field. This is true, because Terfenol-D's magnetic permeability, and thus its corresponding blocked inductance, is significantly smaller than Galfenol's. Larger wire diameters (smaller wire gauges) also provide an improvement in the rise time, because they can carry larger currents; consequently, they can generate $H_{\text {max }}$ with fewer windings and thus a less inductive electromagnet. Fig. 1b depicts the average electrical power required to reach $H_{\max }$ for each design case. Power demands are essentially independent of the rod's length and the material. Unsurprisingly, the improvement in rise time achieved by using larger currents comes at the expense of increased power demand from the electrical amplifier. 


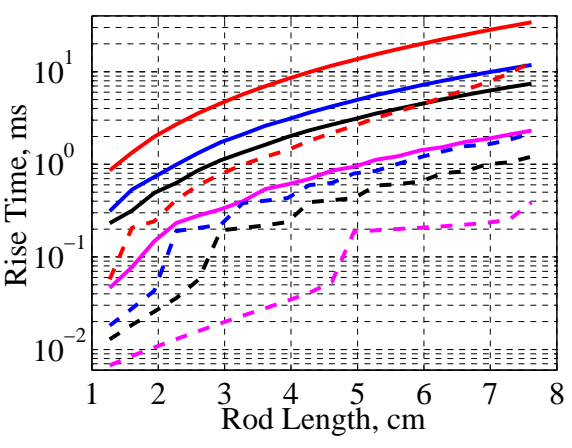

(a)

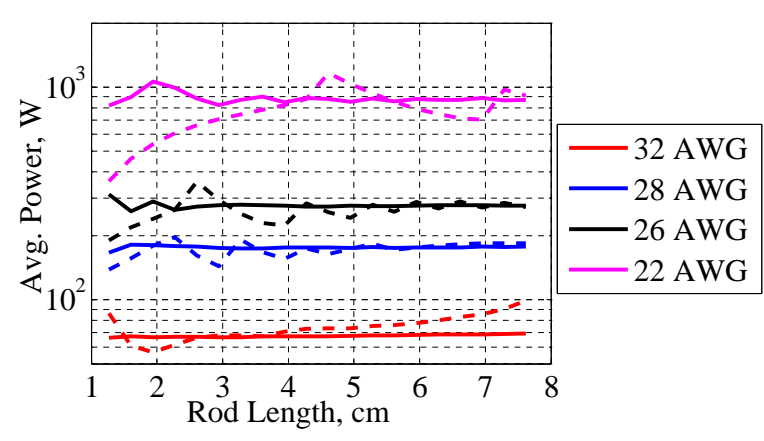

(b)

Figure 1: Electrical response of the Varispring for $m=2 \mathrm{~kg}, E^{H}=E_{\mathrm{sat}}$ (the saturation Young's modulus), and electromagnets wound with $32,28,26$, and 22 AWG wire, (a) rise time to reach $H_{\max }$ from $H=0$ in response to a $250 \mathrm{~V}$ step voltage input and (b) average electrical power required to reach $H_{\text {max }}$; Galfenol (solid), Terfenol-D (dashed).

\subsection{Effect of Dynamic Stress on Bias Magnetic Fields}

When magnetostrictive rods are subjected to constant surface magnetic fields and dynamic axial stresses, eddy currents are generated internally such that magnetic flux changes inside the rod are attenuated. ${ }^{22}$ This effect has been termed mechanically-induced magnetic diffusion. The effect causes a dynamic magnetic field inside the rod and a reduction of the rod's stiffness tunability. Eddy currents should therefore be minimized by keeping the stress frequency low and laminating the rod. Scheidler and Dapino ${ }^{22}$ derived a cut-off frequency $\omega_{c}$ for this effect as the frequency for which the dynamic magnetic flux density at the rod's axis has an amplitude of $\left(J_{0}(\sqrt{-1})\right)^{-1} \approx 0.7898$ times that at the rod's surface,

$$
\omega_{c}=4.3393\left(\mu \sigma R^{2}\right)^{-1},
$$

where $R$ and $\sigma$ are the radius and electrical conductivity of the rod, respectively. Eq. 7 is valid for solid rods operating in linear regimes. The cut-off frequency is plotted as a function of the magnetostrictive rod's length in Fig. 2, using the properties listed in Table 1. The cut-off frequency of the Terfenol-D rod is over two orders of magnitude greater than that of the Galfenol rod. Therefore, to get the same flux penetration at a given frequency, the Galfenol rod would need to be much more finely laminated than the Terfenol-D rod. However, there is a practical limit on the thickness of laminates due to manufacturing capabilities. Further, the presence of glue between laminates reduces the stiffness and stiffness tunability of the rod.

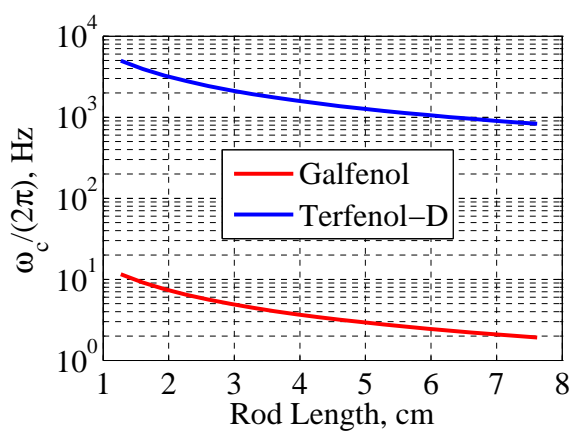

Figure 2: Cut-off frequency for mechanically-induced magnetic diffusion in the magnetostrictive rod. 


\subsection{Effect of Internal Mass on Dynamic Stiffness}

It is well known that the mass of a structure influences its dynamic stiffness and that this effect increases with frequency. The objective of the experimental testing of the Varispring was to measure the changes in the device's stiffness caused by changes in the elastic component of the magnetostrictive rod's stiffness. Changes in stiffness due to mass effects would have corrupted the experimental results. Consequently, the rod was designed to minimize these effects.

Below the first mechanical resonance, the effect of internal mass $m=\rho A_{\text {rod }} l_{\text {rod }}$ on the dynamic stiffness $D$ of the magnetostrictive rod can be approximated using the lumped parameter model shown in Fig. 3a, where $\rho$ is the density. For this model, the driving-point stiffnesses $D_{11}, D_{22}$ and cross-point stiffnesses $D_{12}$, $D_{21}$ are

$$
D_{11}(\omega)=D_{22}(\omega)=\frac{2 K\left(2 K-m \omega^{2}\right)}{4 K-m \omega^{2}}, D_{12}(\omega)=D_{21}(\omega)=\frac{-4 K^{2}}{4 K-m \omega^{2}},
$$

where $\omega$ is the forcing frequency. Fig. 3b depicts the absolute value of the percent change in the driving-point and cross-point stiffnesses of the Galfenol and Terfenol-D rods for worst case conditions (i.e., the maximum frequency, $1000 \mathrm{~Hz}$, and minimum expected Young's modulus of the materials $E_{\min }^{H}$ ) using properties from Table 1. The effect of mass on the dynamic stiffness of the Terfenol-D rod is nearly double that of the Galfenol rod; however, the effect is small in both rods for the parameters considered.

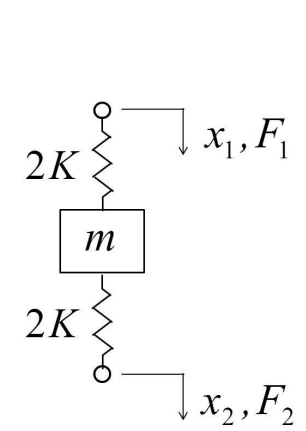

(a)

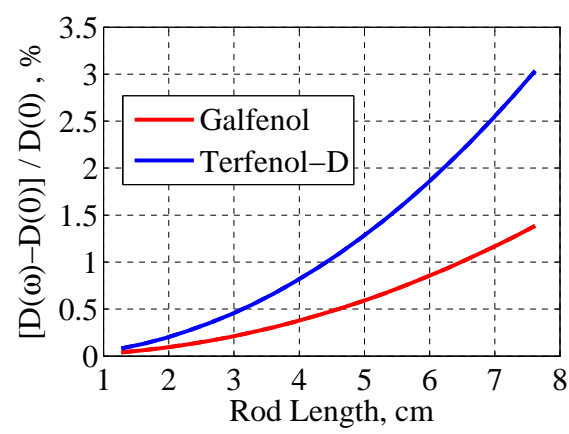

(b)

Figure 3: Effect of internal mass on the dynamic stiffness of the magnetostrictive rod, (a) lumped parameter model of the rod and (b) absolute value of the percent change in the driving-point and cross-point dynamic stiffnesses from their static values for worst case conditions $\left(E^{H}=E_{\min }^{H}\right.$ and $\left.\omega=2 \pi 1000 \mathrm{rad} / \mathrm{s}\right)$, drivingpoint and cross-point stiffnesses overlap.

\subsection{Varispring Design}

Terfenol-D was selected as the magnetostrictive material, because (a) a Terfenol-D-based Varispring has a rise time almost an order of magnitude faster than a Galfenol-based Varispring, (b) its diffusion cut-off frequency is over two orders of magnitude greater than that of Galfenol for the same geometry, and (c) it exhibits a considerably larger variation in quasi-static elastic modulus than Galfenol. ${ }^{12,18}$ The Terfenol-D $\left(\mathrm{Tb}_{0.3} \mathrm{Dy}_{0.7} \mathrm{Fe}_{1.92}\right)$ rod was purchased from Etrema Products, Inc. To improve the dynamic performance of the Varispring, the Terfenol-D was laminated with $0.762 \mathrm{~mm}$ (0.030 in) laminations and adhesive layers of about $0.048 \mathrm{~mm}(0.0019 \mathrm{in})$. The elastic modulus of the adhesive is $862 \mathrm{MPa}(125 \mathrm{ksi})$. The analysis in the preceding sections shows that a shorter Terfenol-D rod provides better performance. However, the rod had to be long enough to attach sensors to it. A length of $2.401 \mathrm{~cm}(0.9453 \mathrm{in})$ was selected to balance these criteria. A diameter of $1.271 \mathrm{~cm}$ (0.5005 in) provided the desired maximum axial stiffness of the rod.

A CAD model of the prototype Varispring is shown in Fig. 4a. The central load path - from the input through the Terfenol-D rod and along the device's cylindrical axis to the output - acts as an elastic member 
(i.e., a spring) with a variable stiffness. Stiffness is modulated via changes in the electromagnet's current and thus, the axial magnetic field applied to the Terfenol-D. The magnetic flux return path ensures that Terfenol-D's magnetic state is homogeneous under quasi-static operating conditions (i.e., in the absence of magnetic diffusion). A sleeve bushing isolates the flux return path from forces applied to the central load path. The flux return path also counteracts the bias load, which was applied by a Century Spring CDM401413 Belleville spring. A non-magnetic bottom cover contains a Kistler 9001A piezoelectric load washer, which was used to measure the axial, dynamic force. Blind holes on the ends of the Varispring were used to align the device to the load frame during testing. The electromagnet was wound using 519 turns of 22 AWG wire and was held together by Duralco 4525 epoxy having a thermal conductivity of $1.875 \mathrm{~W} /\left(\mathrm{m}{ }^{\circ} \mathrm{K}\right)$.

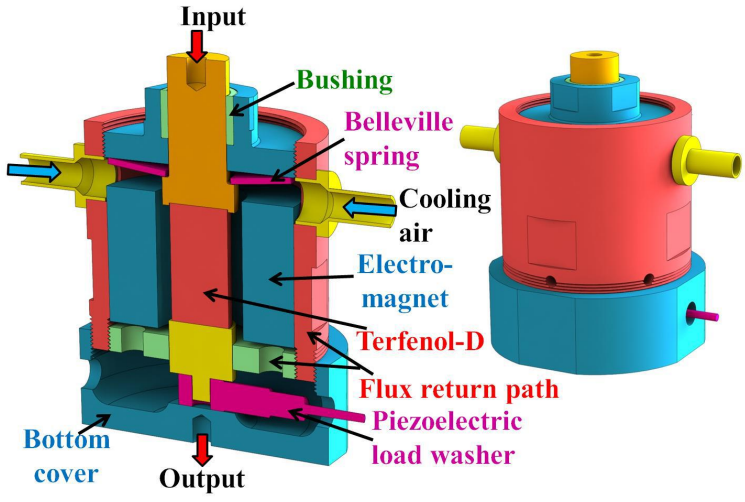

(a)

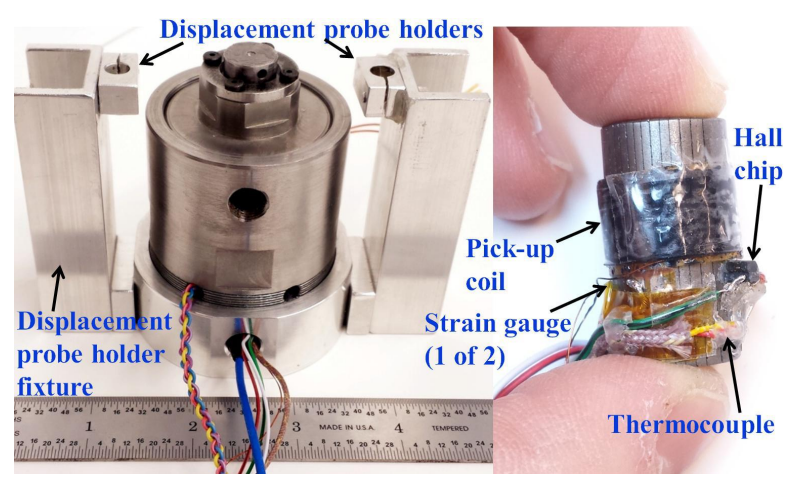

(b)

Figure 4: (a) CAD model of the prototype Varispring and (b) manufactured Varispring (air hose fittings removed) with capacitive displacement probe holder fixture attached (left) and Terfenol-D rod with sensors attached (right); the material of every passive structural component is 1018 steel, except for the Belleville spring, bushing, air hose fittings, and bottom cover, which are high carbon steel, Rulon J, brass, and 7075 $\mathrm{Al}$, respectively.

Using equations given by Scheidler et al., ${ }^{18}$ the inertial force error at $1000 \mathrm{~Hz}$ for this design is about $0.2 \%$. The stiffness ratio of the central load path to the flux return path is approximately $0.3 \%$, primarily due to the relatively high compliance of the Belleville spring; thus, when the Varispring was assembled, the Belleville spring compressed to near maximum deflection as desired.

The manufactured prototype Varispring and Terfenol-D rod are shown in Fig. 4b. The U-channel fixtures on the sides of the device held MicroSense 8810 capacitive displacement probes, which measured the total displacement of the Varispring, i.e., the relative displacement between the bottom of the Varispring and a cylindrical target (depicted in Fig. 5) that mounted to the top of the device. The displacement probe target and displacement probe holder fixture were designed to be nearly rigid up to $1000 \mathrm{~Hz}$. The first natural frequency of the target and fixture were calculated as 5541 and $1492 \mathrm{~Hz}$, respectively, using COMSOL Multiphysics.

\section{EXPERIMENTAL RESULTS}

Experimental testing was conducted using an MTS 831.50 high frequency load frame. The axial strain at the surface of the Terfenol-D rod was measured using a pair of Vishay Micro-Measurements EA-06250BF-350/L strain gauges, which were bonded axially on opposite sides of the rod and wired in series to cancel electromagnetic noise induced in the gauges due to the time-varying magnetic flux density in the rod. ${ }^{18}$ Temperature was measured using a Type $\mathrm{K}$ thermocouple to ensure that temperature variations were minimal. Magnetic flux density was measured with a Lake Shore Model 480 fluxmeter and custom pick-up coil. An Allegro A1302ELH Hall effect sensor was utilized to measure the axial magnetic field at the surface of the Terfenol-D rod. The electrical excitation was generated by a Techron LVC 5050 linear 
amplifier operated in current control mode. The voltage supplied to the Varispring was measured using the amplifier's voltage monitor, while the current supplied to the device was calculated from the voltage drop across a $0.1 \pm 0.001 \mathrm{Ohm}, 15 \mathrm{~W}$ Leeds \& Northrup Co. resistor connected in series with the Varispring. The amplifier's current monitor was not used, because it exhibited an erroneous offset that changed throughout the experiment. The sensors were calibrated as described by Scheidler et al., ${ }^{18}$ except for the pick-up coil, which was calibrated by measuring the static magnetic field generated in air by a large electromagnet using the calibrated Hall sensor and pick-up coil. Signals were phase aligned in post processing by correcting for the phase response of the conditioning electronics. ${ }^{18}$

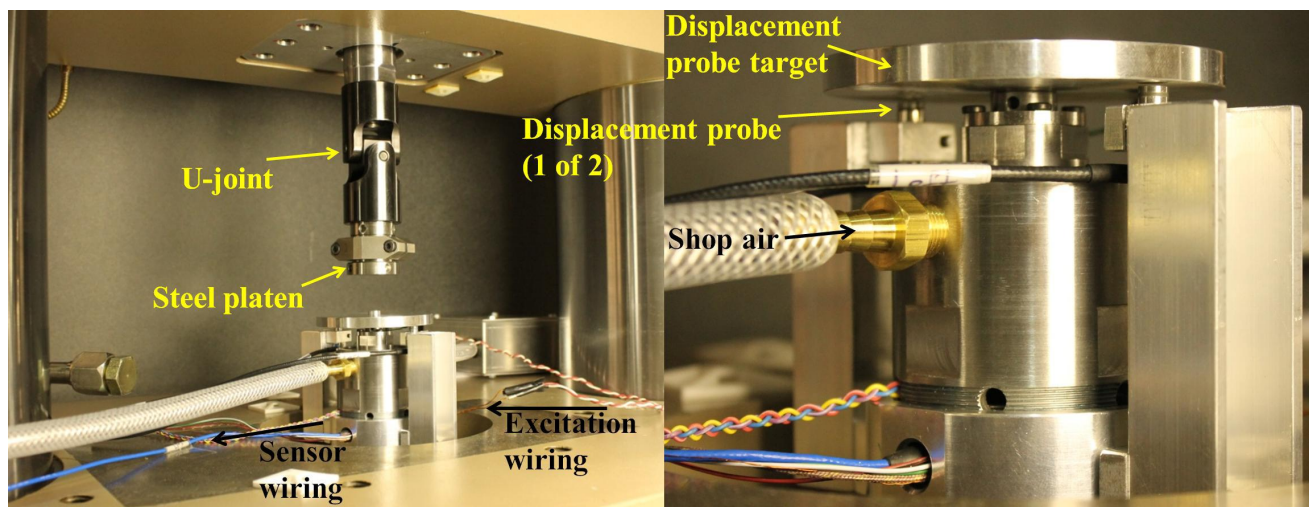

Figure 5: Experimental setup (the compression spring used to improve the force control is not shown).

Fig. 5 shows the experimental setup. Initially, force was applied to the Varispring by directly engaging the displacement probe target with the steel platen. However, for this setup, force control was poor due to the large, high-speed stiffness changes of the Varispring (i.e., a disturbance to the force control system). Force control was greatly improved by placing a soft compression spring between the steel platen and displacement probe target. This isolation spring acted as a mechanical low-pass filter to attenuate the disturbance. The performance of the force control when using the soft spring is depicted in Fig. 6. For all cases except the $1000 \mathrm{~Hz}$ sinusoidal current, the force control performs very well. However, due to the large motion of the steel platen that occurred when the soft spring was used, the displacement probes were removed from the experimental setup to prevent the possibility of damaging the probes. Thus, the stiffness of the Varispring could not be calculated in this paper; this issue will be resolved in future work. Here, the strain response and Young's modulus of the Terfenol-D rod were used to investigate the change in the Varispring's elastic state.

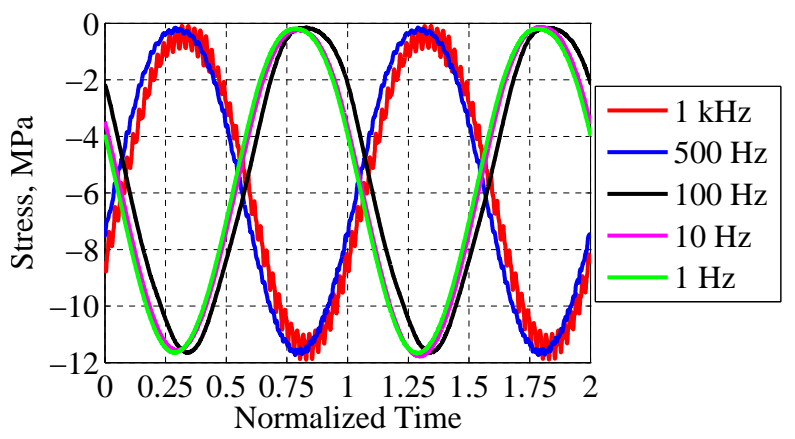

Figure 6: Force control performance when using the soft compression spring; $6 \mathrm{MPa}$ amplitude, $25 \mathrm{~Hz}$ sinusoidal forcing and 1.5 A amplitude sinusoidal current of varying frequency. 


\subsection{Dynamic Stiffness Tuning}

Preliminary measurements of the dynamic stiffness tuning behavior of the Varispring were obtained by measuring the strain response to a 2.00 (or 6.00) MPa amplitude, $25 \mathrm{~Hz}$ sinusoidal stress while continuously varying the stiffness via a 1 to $1000 \mathrm{~Hz}$ sinusoidal current. The current had a nominal amplitude and bias of $1.50 \mathrm{~A}$; these conditions were selected, because an earlier measurement of the quasi-static stiffness at a bias stress of $-25.5 \mathrm{MPa}$ found that the maximum stiffness change occurred as current varied from 0.00 to 3.08 A. For dynamic stiffness tuning, the isolation spring was used to improve load control. Consequently, the magnitude of the bias compression was decreased from 25.5 MPa to 5.90 $\mathrm{MPa}$ to prevent full compression of the isolation spring.

Fig. 7 depicts the strain response of the Terfenol-D rod inside the prototype Varispring for $1 \mathrm{~Hz}$ and 100 $\mathrm{Hz}$ sinusoidal currents. The strain response due to the $25 \mathrm{~Hz}$ forcing is superimposed on a response having the same frequency as the current, because the current actuates the Terfenol-D rod in addition to varying its elastic properties. The Young's modulus of the rod modulates between a soft and a stiff value, indicated by the red and blue annotations, respectively. At a bias of $-5.90 \mathrm{MPa}$, the difference between the stiff and soft modulus for the $1 \mathrm{~Hz}$ current is $20 \mathrm{GPa}$, which is significantly smaller than the difference measured during static stiffness tuning at a bias of $-25.5 \mathrm{MPa}$ (about 68 to $18 \mathrm{GPa}$, or a $50 \mathrm{GPa}$ difference). This disparity primarily results from the change in bias stress. Thus, a large improvement in the dynamic stiffness tuning range can be achieved by improving the experimental setup such that the Varispring can be accurately tested at a more optimal bias stress; this can be realized by using an isolation spring with a larger stroke. Table 2 summarizes the approximate high and low Young's modulus for each loading case. The amplifier was unable to maintain a consistent current amplitude for each case, although the same current control voltage input was used. This is the primary source of variation in the moduli. Despite this issue, the observed stiffness tuning range is relatively constant up to $500 \mathrm{~Hz}$, after which the tuning range significantly decreases.

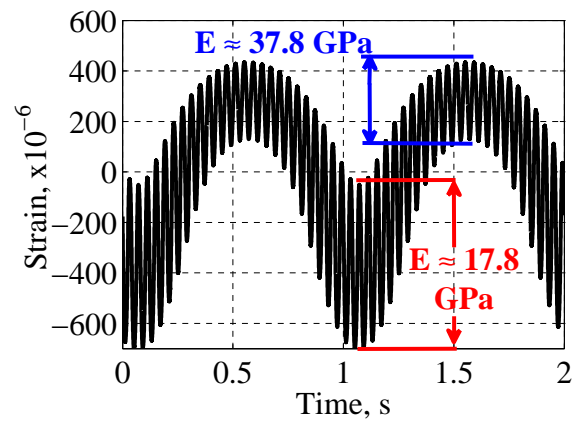

(a)

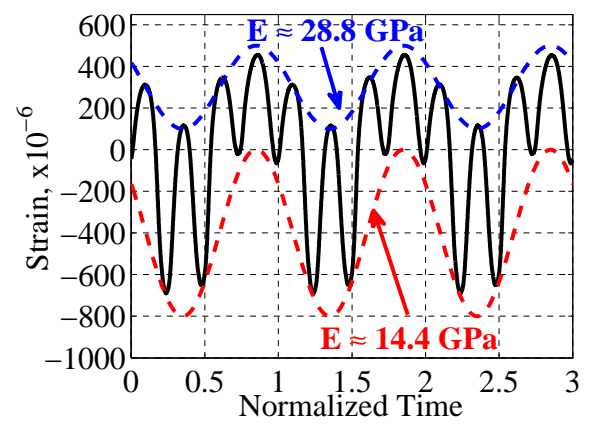

(b)

Figure 7: Strain response of the Terfenol-D rod inside the prototype Varispring to a $25 \mathrm{~Hz}, 6 \mathrm{MPa}$ amplitude stress and $-5.90 \mathrm{MPa}$ bias, (a) $1 \mathrm{~Hz}$ current and (b) $100 \mathrm{~Hz}$ current.

Table 2: Approximate low and high Young's moduli of the Terfenol-D rod inside the prototype Varispring at different current frequencies and stress amplitudes for a bias stress of -5.90 MPa.

\begin{tabular}{c|ccc|ccc}
\hline $\begin{array}{c}\text { Frequency, } \\
\mathrm{Hz}\end{array}$ & Low, GPa & $\begin{array}{c}2 \mathrm{MPa} \\
\text { High, GPa }\end{array}$ & $\Delta, \mathrm{GPa}$ & Low, GPa & High, GPa & $\Delta, \mathrm{GPa}$ \\
\hline 1 & 25.5 & 42.1 & 16.6 & 17.8 & 37.8 & 20.0 \\
100 & 15.0 & 34.5 & 19.5 & 14.4 & 28.8 & 14.4 \\
500 & 13.9 & 35.8 & 21.9 & 15.3 & 29.8 & 14.5 \\
1000 & 22.0 & 26.8 & 4.80 & 19.9 & 22.8 & 2.90 \\
\hline
\end{tabular}




\section{SUMMARY AND CONCLUSIONS}

This paper detailed the development of a magnetostrictive transducer designed to operate as a spring element with a dynamically-tunable and electrically-controllable stiffness. This device was termed a magnetostrictive Varispring. The Varispring can be applied as a semi-active vibration isolator or switched stiffness vibration controller for reducing transmitted vibrations. The Varispring was designed by modeling (a) the coupled electrical response using a linear transducer model, (b) the effect of dynamic stress on bias magnetic fields (mechanically-induced magnetic diffusion), and (c) the effect of internal mass on the dynamic stiffness of the active element. Experimental and practical design considerations were also discussed.

The theoretical performance of Galfenol- and Terfenol-D-based Varisprings was calculated and compared. For a given wire gauge used to wind the electromagnet, a Terfenol-D-based Varispring has a rise time almost an order of magnitude smaller than a Galfenol-based Varispring, despite the Terfenol-D requiring a much larger magnetic field for stiffness tuning. The cut-off frequency for mechanically-induced magnetic diffusion in the Terfenol-D rod is over two orders of magnitude greater than that of the Galfenol rod. Therefore, to get the same flux penetration at a given frequency, the Galfenol rod would need to be much more finely laminated than the Terfenol-D rod. The effect of internal mass on the dynamic stiffness of both rods is below $3 \%$ for the parameters considered. These modeling results motivated the use of a laminated Terfenol-D rod for a greater stiffness tuning range and increased bandwidth.

A prototype Varispring was manufactured and then tested under vibratory excitation using a dynamic load frame. For this prototype, the stiffness was indirectly varied by controlling the excitation current, as opposed to direct variation through control of the magnetic field. Preliminary measurements of the dynamic stiffness tuning behavior of the Varispring were obtained by measuring the strain response to a 2.00 (or 6.00) $\mathrm{MPa}$ amplitude, $25 \mathrm{~Hz}$ sinusoidal stress while continuously varying the stiffness via a 1 to $1000 \mathrm{~Hz}$ sinusoidal current. The Young's modulus of the Terfenol-D rod inside the Varispring can be continuously varied by up to $21.9 \mathrm{GPa}$ at a bias stress of $-5.90 \mathrm{MPa}$. The observed stiffness tuning range is relatively constant up to 500 $\mathrm{Hz}$, but significantly decreases thereafter. The stiffness tuning range can be greatly increased by improving the current and force control such that a more consistent current can be applied and the Varispring can be accurately tested at a more optimal bias stress.

\section{ACKNOWLEDGMENTS}

This work was supported by the NASA Aeronautics Scholarship Program (grant \# NNX14AE24H). Additional support was provided by the NASA Aeronautics Research Mission Directorate Seedling Fund and the Smart Vehicle Concepts Center (www.SmartVehicleCenter.org), a National Science Foundation Industry/University Cooperative Research Center.

\section{REFERENCES}

[1] Scheidler, J. and Dapino, M., "Nonlinear dynamic modeling and resonance tuning of Galfenol vibration absorbers," Smart Materials and Structures 22(8), 085015 (2013).

[2] Kellogg, R. and Flatau, A., "Wide band tunable mechanical resonator employing the $\delta \mathrm{E}$ effect of Terfenol-D," Journal of Intelligent Material Systems and Structures 15(5), 355-368 (2004).

[3] Flatau, A. B., Dapino, M. J., and Calkins, F. T., "High bandwidth tunability in a smart vibration absorber," Journal of Intelligent Material Systems and Structures 11(12), 923-929 (2000).

[4] Pagliarulo, P., Kuhnen, K., May, C., and Janocha, H., "Tunable magnetostrictive dynamic vibration absorber," in [Proceedings of the 9th International Conference on New Actuators], 367-370 (2004).

[5] Wright, R. and Kidner, M., "Vibration absorbers: a review of applications in interior noise control of propeller aircraft," Journal of Vibration and Control 10(8), 1221-1237 (2004).

[6] Scheidler, J. J. and Dapino, M. J., "Stiffness tuning of FeGa structures manufactured by ultrasonic additive manufacturing," in [Proceedings of SPIE], 9059, 905907 (2014).

[7] Clark, W. W., "Vibration control with state-switched piezoelectric materials," Journal of Intelligent Material Systems and Structures 11(4), 263-271 (2000).

[8] Ramaratnam, A. and Jalili, N., "A switched stiffness approach for structural vibration control: theory and real-time implementation," Journal of Sound and Vibration 291(1), 258-274 (2006). 
[9] Clark, A. and Savage, H., "Giant magnetically induced changes in the elastic moduli in Tb0.3Dy0.7Fe2," IEEE Transactions on Sonics and Ultrasonics 22(50) (1975).

[10] Savage, H., Clark, A., and Powers, J., "Magnetomechanical coupling and $\delta \mathrm{E}$ effect in highly magnetostrictive rare earth-Fe 2 compounds," IEEE Transactions on Magnetics 11(5), 1355-1357 (1975).

[11] Moffett, M. B., Clark, A. E., Wun-Fogle, M., Linberg, J., Teter, J. P., and McLaughlin, E. A., "Characterization of Terfenol-D for magnetostrictive transducers," The Journal of the Acoustical Society of America 89(3), 14481455 (1991).

[12] Kellogg, R. and Flatau, A., "Experimental investigation of Terfenol-D's elastic modulus," Journal of Intelligent Material Systems and Structures 19(5), 583-595 (2008).

[13] Kellogg, R. A., Flatau, A. B., Clark, A. E., Wun-Fogle, M., and Lograsso, T. A., "Quasi-static transduction characterization of Galfenol," Journal of Intelligent Material Systems and Structures 16, 471 (2005).

[14] Atulasimha, J., Flatau, A. B., and Kellogg, R. A., "Sensing behavior of varied stoichiometry single crystal Fe-Ga," Journal of Intelligent Material Systems and Structures 17(2), 97-105 (2006).

[15] Wun-Fogle, M., Restorff, J., and Clark, A., "Soft and hard elastic moduli of Galfenol transduction elements," Journal of Applied Physics 105(7), 07A923-07A923 (2009).

[16] Datta, S., Atulasimha, J., Mudivarthi, C., and Flatau, A., "Stress and magnetic field-dependent Young's modulus in single crystal iron-gallium alloys," Journal of Magnetism and Magnetic Materials 322(15), 2135-2144 (2010).

[17] Restorff, J., Wun-Fogle, M., and Summers, E., "Hysteresis, $d *_{33}$ and $d_{33}$ of Fe 81.6 Ga $_{18.4}$ textured polycrystals," Journal of Applied Physics 109(7), 07A922 (2011).

[18] Scheidler, J., Asnani, V., and Dapino, M., "Dynamic characterization of Galfenol (Fe81.6 Ga18.4)." NASA Technical Publication (in review).

[19] ASTM International, "ASTM E 467 - 08: Standard practice for verification of constant amplitude dynamic forces in an axial fatigue testing system," (2008).

[20] Hunt, F. V., [Electroacoustics: the analysis of transduction, and its historical background], Acoustical Society of America (1954).

[21] Engdahl, G., ed., [Handbook of Giant Magnetostrictive Materials], Academic Press, San Diego, CA (2000).

[22] Scheidler, J. and Dapino, M., "Mechanically-induced magnetic diffusion in cylindrical ferromagnets," Journal of Magnetism and Magnetic Materials (in review). 